\title{
ESTIMATING SPACE-VARIANT MOTION BLUR WITHOUT DEBLURRING
}

\author{
Shengyang Dai and Ying Wu \\ Department of EECS, Northwestern University \\ 2145 Sheridan Road, Evanston, IL 60208 \\ \{sda690,yingwu\}@ece.northwestern.edu
}

\begin{abstract}
Identifying space-variant motion blurs is a very challenging task in blind blur identification research. This paper describes a novel method towards blind identification without deblurring. Based on the image gradients in the $\alpha$-channel component of a blurred color image, an elegant $\alpha$-motion blur constraint is proposed, which is a linear constraint for local motion blur parameters. It makes possible efficient blind identification of space-variant and even nonlinear motion blurs and the estimation of motion blur fields, without deblurring.
\end{abstract}

Index Terms - space-variant blur, blind identification

\section{INTRODUCTION}

Being a common type of image degradation, motion blur has been widely investigated in image recovery and most of the research assumed linear space-invariant motion blurs. In real applications, however, a degraded picture is likely to present space-variant and nonlinear blurs when the motion becomes more complex than simple 2D translational motion. For example, the image may contain multiple moving objects, or the moving object undergoes 3D motion that largely changes its depth and orientation. Without the prior knowledge of the motion, estimating such complex motion blurs and deblurring the picture is a very challenging task in blind blur identification $(\mathrm{BBI})$ research $[1,2]$.

There have been some attempts to overcome this great challenge in image recovery. An early attempt looked at the zero-crossings in the frequency domain [3,4]. This idea is natural, but it is confronted by the noise in the blurred image, as the true zero-crossings that correspond to the blur cannot be reliably identified from a large number of zero-crossings that are produced by unknown noise. Then the most popular approach in the recent research has been performing blur estimation and deblurring simultaneously $[5,6]$, which is quite computationally demanding as there are a large number of unknowns to be estimated. In addition, complex blurs such as space-variant and nonlinear blurs still remain open.

Can motion blur be identified and estimated without deblurring? If so, deblurring will be significantly less computationally demanding. Very recently, methods that decouples blur identification and deblurring have been suggested. For example, blurs can be identified and estimated locally by analyzing image patches $[7,8,9]$ to enable space-variant deblurring. We propose a novel approach in this paper to give a positive answer to this question on pixel level.

To infer and estimate complex motion blurs, the most important thing is to extract informative image observations that are closely related to the blur and invariant to irrelevant factors, and to construct effective constraints for blur estimation. In this paper, we propose a novel local motion blur constraint, which is based on the image gradient information in the $\alpha$ channel component of a blurred color image. This new constraint is local, as it is pixel-wise. In addition, it is linear with respect to the motion blur parameters. More importantly, this constraint only has two free parameters in general, and in most case they can be further reduced to a binary value $( \pm 1)$. Thus, this new constraint enables elegant and powerful solutions to blind blur identification and estimation.

\section{MOTION BLUR CONSTRAINT}

Before working on discretized case, we first describe our motion blur constraint on the continuous case, where the original non-blurred signal is a continuous function $I$ on $\mathbb{R}^{2}$. I is convolved with an ideal motion blur kernel $h$ (point spread function, or PSF), which is fully characterized by its direction $\theta(\theta \in[0, \pi))$ and length $l(l>0)$. When $\theta=0$, the kernel function is:

$$
h_{0}(x, y)= \begin{cases}\frac{1}{l} \delta(y) & -\frac{l}{2} \leq x \leq \frac{l}{2} \\ 0 & \text { otherwise, }\end{cases}
$$

where $\delta$ is the Dirac delta function. The kernels with nonzero $\theta$ can be obtained by rotating $h_{0}$ with $\theta$. In our work, equivalently, we can parameterize $h$ by two different parameters as a blur vector, $\mathbf{b}=(u, v)^{T}$, where $u=l \cos \theta, v=l \sin \theta$ are the projected lengths of the motion blur kernel on $x$ and $y$ axes, respectively.

For space-invariant motion blur, $\mathbf{b}$ is constant throughout the entire image, and the generation function of the blurred signal is $I_{\mathbf{b}}=I * h$, where $*$ is the convolution operator. We have the following theorem:

Theorem 1 A $2 D$ continuous signal $I$ is motion blurred by kernel $h . I_{\mathbf{b}}=I * h$. The following equation holds at any 


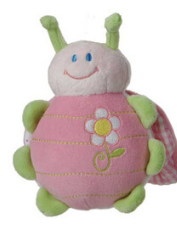

(a)

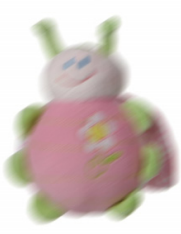

(b)

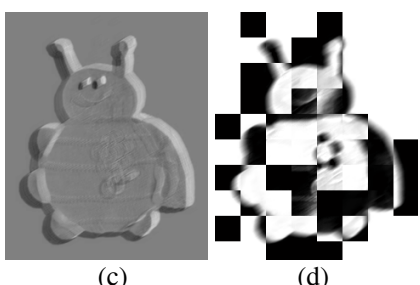

(c)

(d)
Fig. 1. Illustration of motion blur constraint and alpha channel image description: (a) unblurred image (b) motion blurred image (c) $\nabla I_{\mathbf{b}} \cdot \mathbf{b}$ on one color channel (d) alpha channel description of (b).

position $\mathbf{p :}$

$$
\left.\nabla I_{\mathbf{b}}\right|_{\mathbf{p}} \cdot \mathbf{b}=I\left(\mathbf{p}+\frac{\mathbf{b}}{2}\right)-I\left(\mathbf{p}-\frac{\mathbf{b}}{2}\right)
$$

where $\left.\nabla I_{\mathbf{b}}\right|_{\mathbf{p}}=\left.\left(\frac{\partial I_{\mathbf{b}}}{\partial x}, \frac{\partial I_{\mathbf{b}}}{\partial y}\right)^{T}\right|_{\mathbf{p}}$ is the image gradient and $\mathbf{b}=(u, v)^{T}$ is the motion blur vector.

We omit the proof due to the space limit. An illustration on digital image is shown in Fig. 1. Eqn. 2 is verified by Fig. 1(c), which shows a clear pattern of difference between two translated versions of the original image with displacements of $\frac{\mathbf{b}}{2}$ and $-\frac{\mathbf{b}}{2}$.

We call Eqn. 2 the motion blur constraint. For each pixel $\mathbf{p}$, the image gradient of the blurred image $\left.\nabla I_{\mathbf{b}}\right|_{\mathbf{p}}$ can be readily computed. The only two free parameters are two pixels in the unblurred image, rather than an integral over a large number of pixels in the unblurred image. Deblurring is needed to recover these two free parameters, which is the reason that deblurring has to be involved in blur estimation.

On the other hand, if the motion blur constraint has nothing to do with the unblurred image, then blur identification and deblurring can be separated. In the following sections, we describe a novel treatment by using the $\alpha$-channel components to further reduce these two free parameters to one binary unknown, which makes possible the decoupling.

\section{ALPHA CHANNEL IMAGE DECOMPOSITION}

For a color image, image matting is the technique that separates its foreground objects from the background. As the pixels on the object boundary tend to be mixtures of both foreground and background color components, the separation needs to be done softly. A color image can be treated as a linear combination of the foreground and background. Specifically, for each pixel $\mathbf{p}$, we have

$$
I_{\mathbf{p}}=\alpha_{\mathbf{p}} F_{\mathbf{p}}+\left(1-\alpha_{\mathbf{p}}\right) B_{\mathbf{p}}
$$

where $I_{\mathbf{p}}, F_{\mathbf{p}}$, and $B_{\mathbf{p}}$ are the pixel colors of the input, foreground, and background images respectively, $\alpha \in[0,1]$ is the linear combination weight. Alpha matting is the inverse problem of the above generation process. It recovers $F, B$, and $\alpha$ simultaneously for each pixel, given an input color image $I$. An example is shown in Fig. 2

It is clear that image decomposition by alpha matting is severely under-determined, and priors are needed to regularize the problem. One commonly used prior is image smoothness prior, which assumes local color smoothness for both foreground and background. It is extended to local linear color model in [10], resulting in a closed-form solution. User interaction can be considered as another kind of prior, where some pure foreground / background pixels are indicated manually by providing a trimap or using brush tool (Fig. 2(b)).

We rewrite Eqn. 3 as follows

$$
\alpha=\frac{1}{F-B} I-\frac{B}{F-B},
$$

where the position index p in Eqn. 3 is omitted without confusion. Taking the gradient on both sides, based on the local smoothness assumption of $F$ and $B$, we have

$$
\nabla \alpha=\frac{1}{F-B} \nabla I \text {. }
$$

This clearly shows that the edge information is preserved in the $\alpha$-channel. A more important and meaningful view of Eqn. 5 is that the $\alpha$-channel image gradient is a normalized and locally adaptive description of image edge, because $F-B$ in the denominator serves as the normalization factor. Based on Eqn. 4, the magnitude of an image edge is normalized to be a 0 to 1 transition in the $\alpha$-channel. By preserving the edge information, the $\alpha$-channel component of a color image actually provides a large amount of information but in a much simpler form, i.e., a single channel description of a color image. This can greatly simplify many image processing tasks such as image deblurring $[11,12]$ and super-resolution [13].

In addition, using the $\alpha$-channel can explore the relationship among three color channels, and naturally combine all color information from three channels. In fact, color channels are closely related to each other. One example is the linear color model [10] mentioned previously, which means that the pixels colors in a local image patch tends to form a line in the 3D color space. Such information is totally discarded by methods that process three color channels separately. The $\alpha$ channel is extracted in a way that all color information are taken into account simultaneously, thus the color channel relationship information is implicitly integrated.

Traditionally, user interaction is needed to obtain good estimates of the $\alpha$-channel. Very recently, the spectral matting technique [14] has make it fully automatic and unsupervised to decompose a color image into a linear combination of image layers. It can be considered as a generalization of the traditional matting techniques. The foreground / background model is replaced by a multiple layer representation as $I=\sum_{i} \alpha_{i} L_{i}$, where $\sum_{i} \alpha_{i}=1$. In most cases, image pixels have at most two non-zero $\alpha$ values.

\section{4. $\alpha$-MOTION BLUR CONSTRAINT}

Now we show how we can reduce the two free parameters in the motion blur constraint in Eqn 2 to a binary unknown.

From Eqn. 4, based on the local smoothness assumption of $F$ and $B$, the following equation can be easily obtained by 


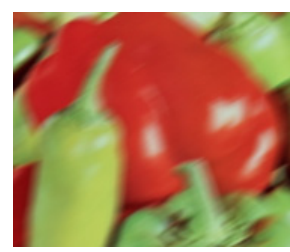

(a)

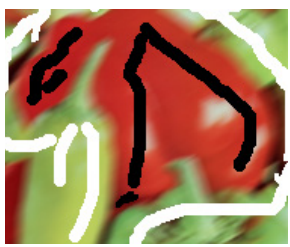

(b)

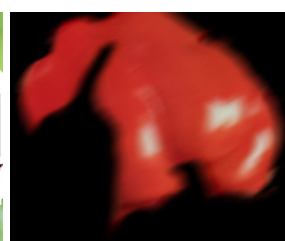

(c)

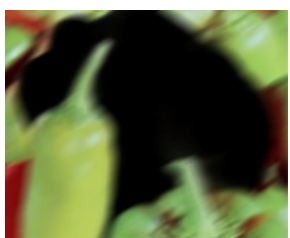

(d)

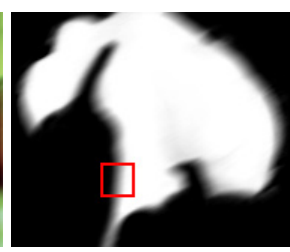

(e)

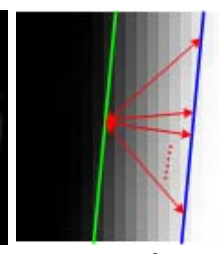

(f)

Fig. 2. Illustration of the alpha matting technique. (a) blurred image, (b) user indication of foreground (in black) and background (in white), (c) foreground component $\alpha F$, (d) background component $(1-\alpha) B$, (e) alpha channel $\alpha$, (f) illustration of Eqn. 8 with a patch of (e).

replacing $I$ with its $\alpha$-channel description $\alpha$ in Eqn. 2:

$$
\left.\nabla \alpha_{\mathbf{b}}\right|_{\mathbf{p}} \cdot \mathbf{b}=\alpha\left(\mathbf{p}+\frac{\mathbf{b}}{2}\right)-\alpha\left(\mathbf{p}-\frac{\mathbf{b}}{2}\right) .
$$

where $\alpha_{\mathbf{b}}=\alpha * h$ is the blurred $\alpha$-channel. We call Eqn. 6 the $\alpha$-motion blur constraint. This constraint suggests that the blur estimation problem for the original color image can be done equivalently in the $\alpha$-channel.

Moreover, working on the $\alpha$-channel enables further reducing the number of free parameter in the motion blur constraint by imposing very mild assumptions. Specifically, if we assume the $\alpha$ values for all pixels in a non-blurred image $I$ are binary, i.e., $\alpha(\mathbf{p}) \in\{0,1\}$, then the uncertainty on the right hand side is largely reduced as it can only be one of three values $\{0,1,-1\}$. Thus, from Eqn. 6 , we have

$$
\nabla \alpha_{\mathbf{b}} \cdot \mathbf{b} \in\{0, \pm 1\}
$$

In reality, this assumption is valid for most pixels in nonblurred images. Due to the high image quality of the modern digital cameras, under good lighting conditions, most image edges are clear and sharp for static objects. Thus almost all pixels in an unblurred image indeed have $\alpha$ values 0 or 1 .

In our experiments, we find that zero in Eqn. 7 is taken mostly when $\nabla \alpha_{\mathbf{b}}=0$. We call pixels satisfying $\left\|\nabla \alpha_{\mathbf{b}}\right\| \neq 0$ valid pixels. Then we can have the following simplified $\alpha$ motion blur constraint hold for each valid pixel:

$$
\nabla \alpha_{\mathbf{b}} \cdot \mathbf{b}= \pm 1
$$

An intuitive explanation of this equation is illustrated by Fig. 2(f). Given a blurred straight edge, the local blur parameters for the center pixel (on the green line) cannot be uniquely determined, since it may correspond to any vector ending on the blur line. That is why the constraint is linear. The magnitude of the blur vector, $\|\mathbf{b}\|$, is proportional to the blur extent, thus inversely proportional to the local $\alpha$-gradient. There is still an ambiguity on the blur direction: $\mathbf{b}$ and $-\mathbf{b}$ can produce the same blurring, which is the uncertainty we have, i.e., \pm 1 in Eqn. 8 .

One important property of the proposed motion blur constraints is that this constraint holds locally for each pixel. In other words, each individual pixel can provide some cues for estimating the local blur parameters. This is very different from most existing motion blur estimation methods, which rely on some global properties, such as finding the zero crossing in frequency domain, matching multiple input images [15], or other coupled blur estimation and recovery method. By integrating such local cues, accurate blur estimation can be obtained in a larger image region. Moreover, by integrating such cues throughout the entire image, blind blur identification and estimation problems can be done for spacevariant and nonlinear motion blurs. In those cases, our motion blur constraint can still be applied because it is local, and the space-variant and nonlinear blurs can be approximated by piece-wise locally invariant linear blurs.

\section{APPLICATION TO MOTION BLUR ESTIMATION}

The above $\alpha$-motion blur constraints are not only simple for estimating space-invariant blurs, but also powerful in identifying and estimating space-variant ones, as the deblurring process is separated and it is no longer involved.

For space-invariant motion blur, a simple voting strategy can be used to estimate the parameters, like the Hough transform. From Eqn. 8, we can see that each valid pixel votes for two lines in the blur parameter space. The final voting result exhibits two peaks in the parameter space, corresponding to two valid blur parameters: $\pm \mathbf{b}$, which cannot be further distinguished, since they can generate the same blurring.

For space-variant motion blur with a global parametric form, e.g., a global rotational motion ${ }^{1}$, we first locally approximate it by a linear form with Taylor expansion. Then we can simply collect the set of the $\alpha$-motion blur constraints for a number of pixels. The set of constraints give an overdetermined linear system, and the blur parameters can be easily estimated by existing estimation techniques such as the Least Square Fitting method.

For multiple unknown motion blurs, the RANSAC technique can be applied to handle multiple models and noises. After estimating those blur models, $\min _{z= \pm 1}\left|\nabla \alpha_{\mathbf{b}} \cdot \mathbf{b}-z\right|$ can be used as the indication of model fitting errors. Combined with a Markov Random Fields model to enforce region continuity and smoothness, blur identification and segmentation can be further obtained. As no prior knowledge is required in this process, it is a blind identification method.

In practice, due to the computational reason, the spectral matting technique [14] is applied independently on overlapping sliding windows of the input blurred image. The

\footnotetext{
${ }^{1}$ In fact, it is both space-variant and nonlinear, since the integral paths for blur generation are curves, instead of straight lines. It has a parametric form, since the blur kernel parameters at each position can be analytically expressed by the rotation parameters.
} 

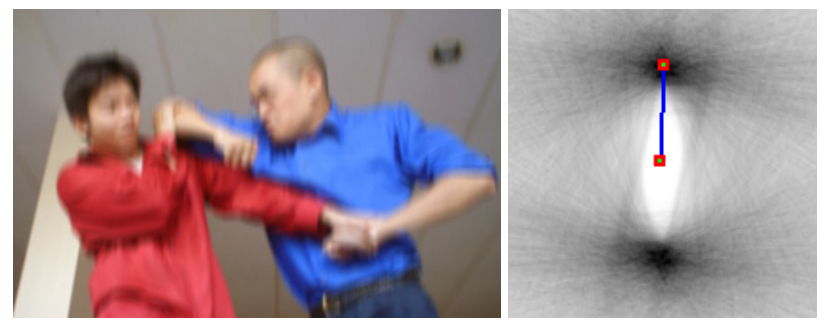

Fig. 3. Space-invariant motion blur estimation. [left]: input image, [right]: voting result in the blur parameter space (i.e. the Hough domain) overlaid with the estimated blur vector.

$\alpha$ value for each pixel is extracted based on the $\alpha$ components for its surrounding window. The $\alpha$ channel gradient $\nabla \alpha=\left(\frac{\partial \alpha}{\partial x}, \frac{\partial \alpha}{\partial y}\right)^{T}$ is computed within each sliding window. The number of matting components in each slicing window is limited to no more than two. Fig. 1(d) gives an example, where the block effect is due to the use of sliding windows.

\section{EXPERIMENTS}

We test our method on real images with various motion blur patterns. Fig. 3 shows an image with space-invariant motion blur. Two points get highest vote on the blur parameter space, corresponding to two plausible blur parameters: $\pm(0.4,12.6)^{T}$. Fig. 4 shows an image with a dominant rotational motion blur, which is space-variant and non-linear. The other part of the image is not blurred and can be treated as outliers and identified by the RANSAC technique. The extracted rotational blur model is visualized in Fig. 4, which clearly shows that our estimation perfectly matches the blur extent of the rotating object. Fig. 5 shows an image where the motion blur is present in only part of the image. The dominant blur parameter, $\mathbf{b}=(17.2,1.4)^{T}$, is estimated by using RANSAC. The model fitting error is visualized. The final segmentation result is obtained by using MRF. These experiments demonstrate the effectiveness of the proposed motion blur constraint. These results are exciting and promising, as the new constraints makes possible the identification and estimation of very complex blur patterns. Space-variant motion deblurring is not discussed here since it is not the main focus, and we leave it as future work.

\section{CONCLUSION}

In this paper, a novel motion blur constraint is proposed by using the $\alpha$-channel components. This linear constraint enables the decoupling of blur estimation and deblurring, and its local property enables estimating various space-variant and nonlinear motion blurs.

Acknowledgments: this work was supported in part by National Science Foundation Grants IIS-0347877 and IIS0308222.

\section{REFERENCES}

[1] Deepa Kundur and Dimitrios Hatzinakos, "Blind image deconvolution,” IEEE Signal Processing Mag., vol. 13, no. 3, pp. 43 - 64, May 1996.

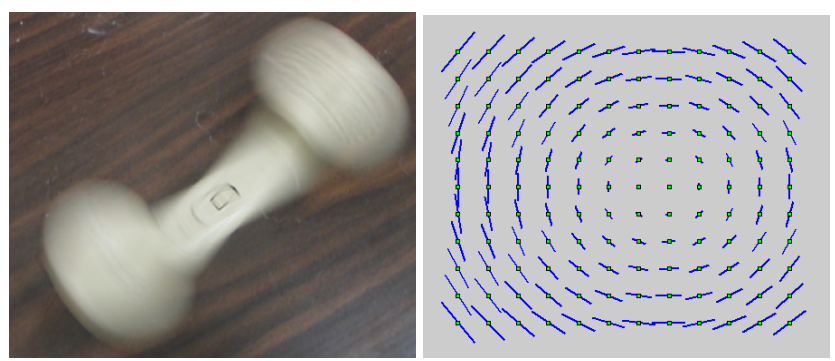

Fig. 4. Rotational blur estimation. [left]: rotational blurred input image, [right]: visualized rotational blur field.
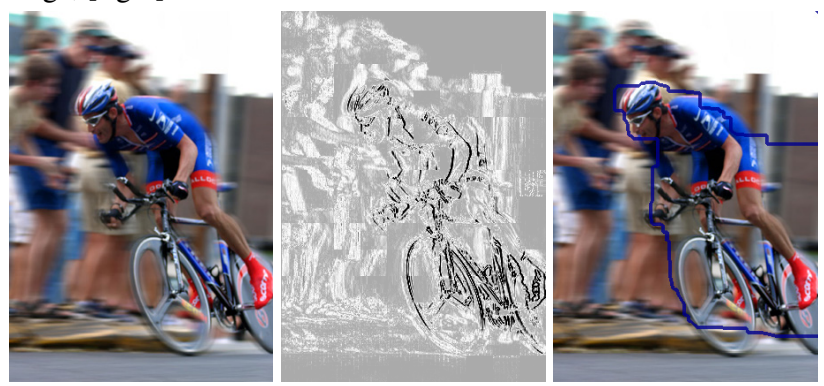

Fig. 5. Local motion blur estimation. [left]: input image, [middle]: visualized model fitting error $\left(\min _{z= \pm 1}\left|\nabla \alpha_{\mathbf{b}} \cdot \mathbf{b}-z\right|\right.$. Zeros are in white, and invalid pixels are in gray. [right]: segmentation result.

[2] Mark R. Banham and Aggelos K.Katsaggelos, "Digital image restoration,” IEEE Signal Processing Mag., vol. 14, no. 2, pp. 24 - 41, Mar. 1997.

[3] Michael Cannon, "Blind deconvolution of spatially invariant image blurs with phase," IEEE Trans. Acoust., Speech, Signal Processing, vol. 24, no. 1, pp. 58 - 63, Feb. 1976.

[4] M.Michael Chang, Murat Tekalp, and A. Tanju Erdem, "Blur identification using the bispectrum," IEEE Trans. Signal Processing, vol. 39, no. 10, pp. 2323 - 2325, Oct. 1991.

[5] S. Derin Babacan, Rafael Molina, and Aggelos K Katsaggelos, "Total variation image restoration and parameter estimation using variational distribution approximation," in $I C I P, 2007$.

[6] Rob Fergus, Barun Singh, Aaron Hertzmann, Sam T. Roweis, and William T. Freeman, "Removing camera shake from a single photograph," ACM Trans. on Graphics (Proc. SIGGRAPH), 2006.

[7] Anat Levin, "Blind motion deblurring using image statistics," in NIPS, 2006.

[8] Shengyang Dai, Ming Yang, Ying Wu, and Aggelos K.Katsaggelos, "Tracking motion-blurred targets in video," in ICIP, 2006.

[9] Ryo Nakagaki and Aggelos K.Katsaggelos, "A VQ-based blind image restoration algorithm," IEEE Trans. Image Processing, vol. 12, no. 9, pp. 1044 - 1053, Sept. 2003.

[10] Anat Levin, Dani Lischinski, and Yair Weiss, "A closed form solution to natural image matting," IEEE Trans. on PAMI, 2007.

[11] Jiaya Jia, "Single image motion deblurring using transparency," in CVPR, 2007

[12] Qi Shan, Wei Xiong, and Jiaya Jia, "Rotational motion deblurring of a rigid object from a single image," in ICCV, 2007.

[13] Shengyang Dai, Mei Han, Wei Xu, Ying Wu, and Yihong Gong, "Soft edge smoothness prior for alpha channel super resolution," in CVPR, 2007.

[14] Anat Levin, Alex Rav-Acha, and Dani Lischinski, "Spectral matting," in $C V P R, 2007$.

[15] Alex Rav-Acha and Shmuel Peleg, "Two motion-blurred images are better than one," Pattern Recognition Letters, 2005. 